\title{
Development of modelling strategies for two-way RC slabs
}

\author{
Jiangpeng Shu ${ }^{\mathrm{a}, *}$, David Fall ${ }^{\mathrm{a}}$, Mario Plos ${ }^{\mathrm{a}}$, Kamyab Zandi ${ }^{\mathrm{a}, \mathrm{b}}$, Karin Lundgren ${ }^{\mathrm{a}}$ \\ ${ }^{a}$ Department of Civil and Environmental Engineering, Chalmers University of Technology, 41296 Gothenburg, Sweden \\ ${ }^{\mathrm{b}}$ Material Group, CBI Swedish Cement and Concrete Research Institute, 50115 Borås, Sweden
}

\section{A R T I C L E I N F O}

\section{Article history:}

Received 26 November 2014

Revised 16 June 2015

Accepted 2 July 2015

\section{Keywords:}

Two-way RC slab

FE analysis

Modelling choices

Capacity

Crack

Load distribution

\begin{abstract}
A B S T R A C T
Analyses of tested two-way reinforced concrete (RC) slabs were carried out with varying modelling choices to develop better modelling strategies. The aim was to study how accurately the response of a slab subjected to bending could be predicted with nonlinear finite element (FE) analysis using three-dimensional (3D) continuum elements, and how the modelling choices might influence the analysis results. The load-carrying capacity, load-deflection response, crack pattern and reaction-force distribution of the two-way slab studied were compared to experimental data available. The influence of several modelling parameters was investigated, including geometric nonlinearity, element properties, concrete model, reinforcement model and boundary condition. The results show the possibility of accurately reflecting the experimental results concerning load-carrying capacity, load-deflection response and crack pattern giving proper modelling choices. Moreover, the reaction force distribution was found to be highly influenced by the stiffness of the supports.
\end{abstract}

(c) 2015 Elsevier Ltd. All rights reserved.

\section{Introduction}

Reinforced concrete (RC) slabs are amongst the most exposed parts in structures such as bridges. Thus, it is critical to be able to accurately assess the load-carrying capacity and response of existing RC slabs. In engineering practice, simplified analytical models are normally used to make structural assessments in an effective way for a large number of load cases. However, the few full-scale tests on real bridges show large load overcapacities compared to conventional assessment, e.g. Plos [1], Sas et al. [2]. Consequently, if improved assessment methods are developed and applied, there are huge possibilities to use these reserves.

Assessment of structural safety and functionality is a step-level procedure, with successively improved evaluation integrated to the decision process, as described in Sustainable Bridges [3]. There, it is also stated that nonlinear finite element (FE) analysis has the highest potential for discovering any additional sources of load-carrying capacity in reinforced concrete bridges. Also Model Code 2010 [4] proposes a "level-of-approximation approach" with progressively refined accuracy of the models and physical parameters used; numerical methods like FE analyses are proposed for the highest levels. Nonlinear FE analysis is well suited to enhanced level assessment, which takes the three-dimensional geometry of the structure and its nonlinear

\footnotetext{
* Corresponding author.

E-mail address: Jiangpeng.shu@chalmers.se (J. Shu).
}

response due to e.g. material plasticity, cracking and large deformations into account. For RC slabs, to be able to reflect various failure modes, three-dimensional (3D) continuum FE models are suitable.

However, nonlinear 3D continuum FE analysis is demanding and requires skilled and experienced structural engineers. Furthermore, such an analysis involves many modelling choices that are decisive for how well the analysis results reflect the response of the real structure.

Consequently, strategies for modelling with 3D continuum elements are needed; efficient and robust numerical models are essential to accurately predict the structural behaviour of RC slabs. The purpose of this study is to contribute to the development of such strategies and to give recommendations regarding modelling choices. These should complement more general guidelines already available in literature such as Hendriks et al. [5] and Fib: Bulletin 45 [6].

The reliability of nonlinear FE analyses depends on modelling choices based on assumptions with regard to such aspects as (i) the nonlinearity of material as well as of geometry, (ii) the properties of finite elements and mesh density, (iii) the interaction between concrete and reinforcement, and (iv) the modelling of boundary conditions. To accurately describe the nonlinear behaviour of RC slabs, FE methods have been investigated since the early 1970s [7]. Crisfield [8] offered a total Lagrange description of geometric nonlinearity, which is useful if rotations and displacements are large and strains are small. Regarding concrete material 


$\begin{array}{ll}\text { Nomenclature } \\ E_{c} \quad \text { elastic modulus of concrete } \\ E_{s} \quad \text { elastic modulus of reinforcement steel } \\ f_{c m} & \text { mean compressive strength of concrete } \\ f_{c t m} & \text { mean tensile strength of concrete } \\ f_{y} & \text { yield strength of reinforcement } \\ \varepsilon_{c 1} & \text { strain of concrete at } f_{c m} \\ P & \text { ultimate load }\end{array}$

$s_{1} \quad$ relative displacement at first stage

$s_{2} \quad$ relative displacement at second stage

$s_{3} \quad$ relative displacement at third stage

$\tau_{\max } \quad$ maximum bond stress

$\tau_{f} \quad$ residual bond stress

$t \quad$ thickness of specimen

$v \quad$ Poisson's ratio nonlinearity, models for the numerical simulation of concrete cracking have traditionally been based on either the smeared crack model [9] or the discrete crack model [10]. In the smeared crack approach, a crack is modelled by modifying the strength and stiffness of concrete and by distributing or "smearing" the dissipated energy of the crack along the finite width of a localization band [11]. This so-called crack band approach [12] is widely adopted as a simple technique eliminating or reducing the sensitivity of numerical results to the mesh size of finite elements in simulations involving strain localization due to softening. In the study by Selby \& Vecchio [13], the lateral expansion effect due to Poisson's ratio was taken into account in the structural model. Two techniques exist for modelling RC structures describing the interaction between concrete and reinforcement: full interaction, or the use of a bond model [14-17]. In the former approach, the reinforcement adds stiffness and strength to the concrete elements in the reinforcement direction, whilst in the latter approach, concrete and reinforcement are modelled separately using an interface layer with a friction model in between. In order to determine the reaction distribution, Belletti et al. [18] carried out an FE analysis of an RC one-way slab using interface elements with high compressive stiffness and low tensile stiffness as part of a support; in this way the effective width of the slab was calculated. All these choices for modelling RC structures are applicable to modelling two-way slabs, but the impact from each has not been systematically studied.

The aim of this study is to examine how accurately the response of a two-way RC slab subjected to bending might be predicted through nonlinear FE analysis using 3D continuum elements. Furthermore, the influence of different modelling choices on the analysis results has been investigated. The load-carrying capacity, load-deflection response, crack patterns and reaction force distribution from FE analysis of a two-way slab under a concentrated load has been compared to corresponding experimental data from Fall et al. [19]. The influence of the following modelling choices has been investigated: geometrical nonlinearity, element properties, material models for concrete, reinforcement (including interaction between reinforcement and concrete), and stiffness of supports. Based on the results of this study, a preliminary modelling strategy and modelling recommendations for a 3D FE analysis using continuum elements are presented.

\section{Experiments of slabs}

Fall et al. carried out a series of tests on two-way slabs, which have been reported in [19]; in this section only a brief description of the experiments is made. The geometry and test set-up were designed so that a flexural failure occurred. In a larger test series, three specimens containing traditional steel bar reinforcement in ordinary concrete were tested. The three specimens were intended to be equal; thus they had the same dimensions and reinforcement arrangements, and the results of the three tests were similar as expected. The specimens were two-way octagonal slabs $(80 \mathrm{~mm}$ in thickness) supported on four edges, each by five rollers, and subjected to a point-load at the centre (see Fig. 1). The loading jack was coupled to a load cell which was placed over a steel plate $(280 \times 280 \times 30 \mathrm{~mm})$. To even out the load, a wood fibre board was placed $(t=12 \mathrm{~mm}$ ) between the steel plate and the slab.

Linear variable differential transformers (LVDTs) were used to measure the deformation of the upper surface of the slabs relative to the floor. The loading was deformation controlled by an LVDT close to the centre of the slab. The $2.4 \mathrm{~m}$ wide octagonal slabs were supported on 20 high-tolerance steel pipe rollers, see Fig. 1 (right). The pipe rollers were used to measure reaction forces by glued strain gangues at two sides. Details of the support system are presented in Fig. 2 (left).

To characterise the material, the compressive strength $\left(f_{c m}=50.9 \mathrm{MPa}\right)$ and tensile strength $\left(f_{c t m}=2.7 \mathrm{MPa}\right)$ of the concrete, together with the tensile strength of steel reinforcement $\left(f_{y}=550 \mathrm{MPa}, E_{s}=210 \mathrm{GPa}\right)$ were tested. To provoke load redistribution after cracking, the reinforcement amount was twice as large in one direction as in the other. The reinforcement bars had a diameter of $6 \mathrm{~mm}$, placed with a clear cover of $20 \mathrm{~mm}$ from the bottom of the slab to the denser bottom layer; the layout is illustrated in Fig. 2 (right).

\section{Numerical models}

The finite element software DIANA 9.4.4 was used to model the slab, using a 3D model using solid elements, as displayed in Fig. 3. Due to symmetry and to reduce the computation time, only a quarter of the slab was included in the model. In the FE Model, the upper steel plates of the roller supports (see Fig. 2) were included and interface elements were used between the concrete and steel plates to describe the friction. To model the boundary conditions provided by the roller supports, the translation of the nodes under the steel plates were fixed in both vertical direction and horizontally along the rollers, but free to move in the rolling direction. The translation of all nodes at the symmetry faces were fixed in the perpendicular direction. The jagged skew edge, which was induced because of the way of meshing, was also checked and proven not to attract stress since it was at the free edge.

The loading system, including steel and wood plates, was also modelled using solid and interface elements (see Fig. 3). The load was applied as a prescribed vertical displacement to the centre node at the top of the loading steel plate. To model the distributed load from the hydraulic jack, all the nodes on the top of the steel plate were tied to the centre node so that they had the same vertical deflection. An incremental static analysis was performed using specified increment sizes. Each increment was equivalent to a vertical displacement of $0.1 \mathrm{~mm}$ until the deflection became $3 \mathrm{~mm}$. For larger mid-span deflections, the increments were increased to $1 \mathrm{~mm}$ to save computation time. The analyses were carried out using a regular Newton-Raphson iteration method based on force and energy convergence criteria, with a tolerance 

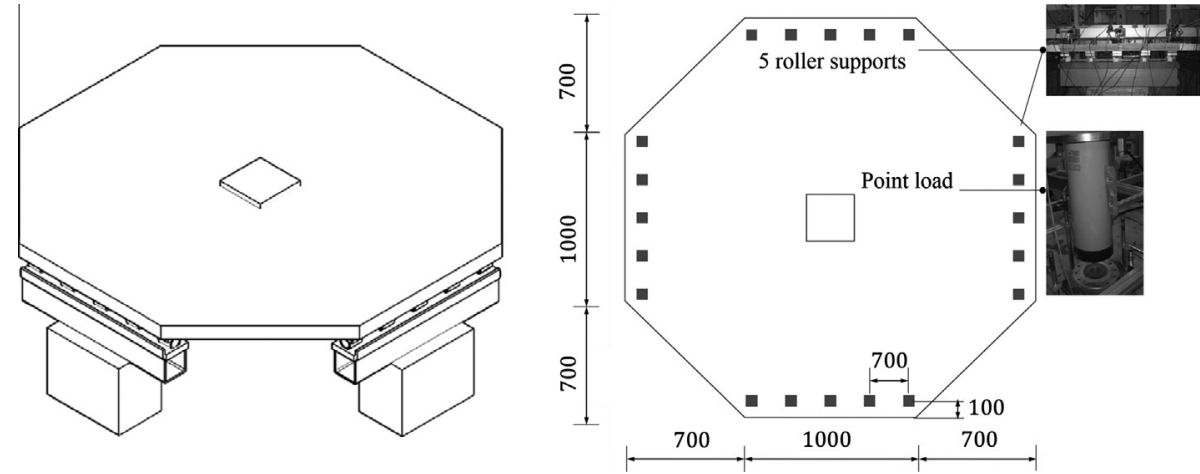

Fig. 1. Test set up of two-way slabs (left) and layout of pipe roller supports (right); measurements in mm [19].
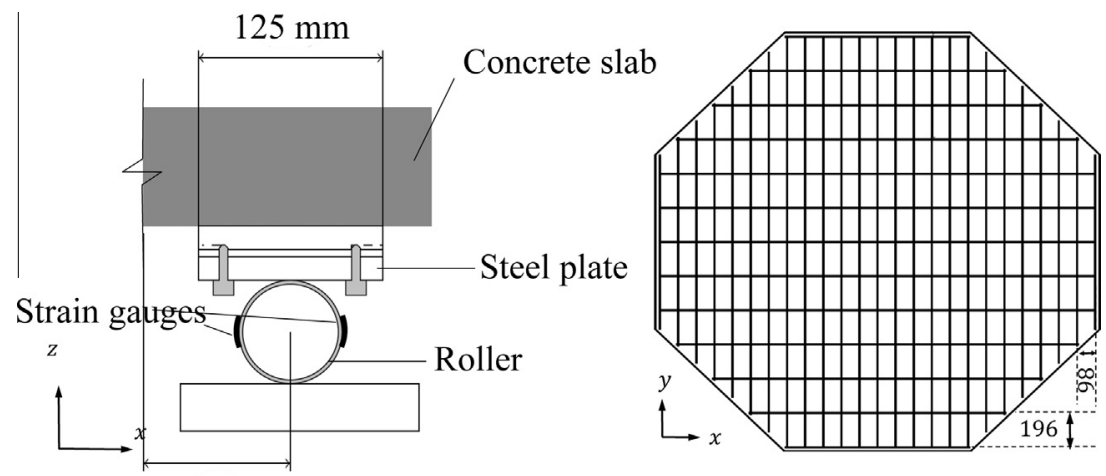

$100 \mathrm{~mm}$

Fig. 2. Support system with pipe roller (left) and reinforcement layout (right), measurements in $\mathrm{mm}$.

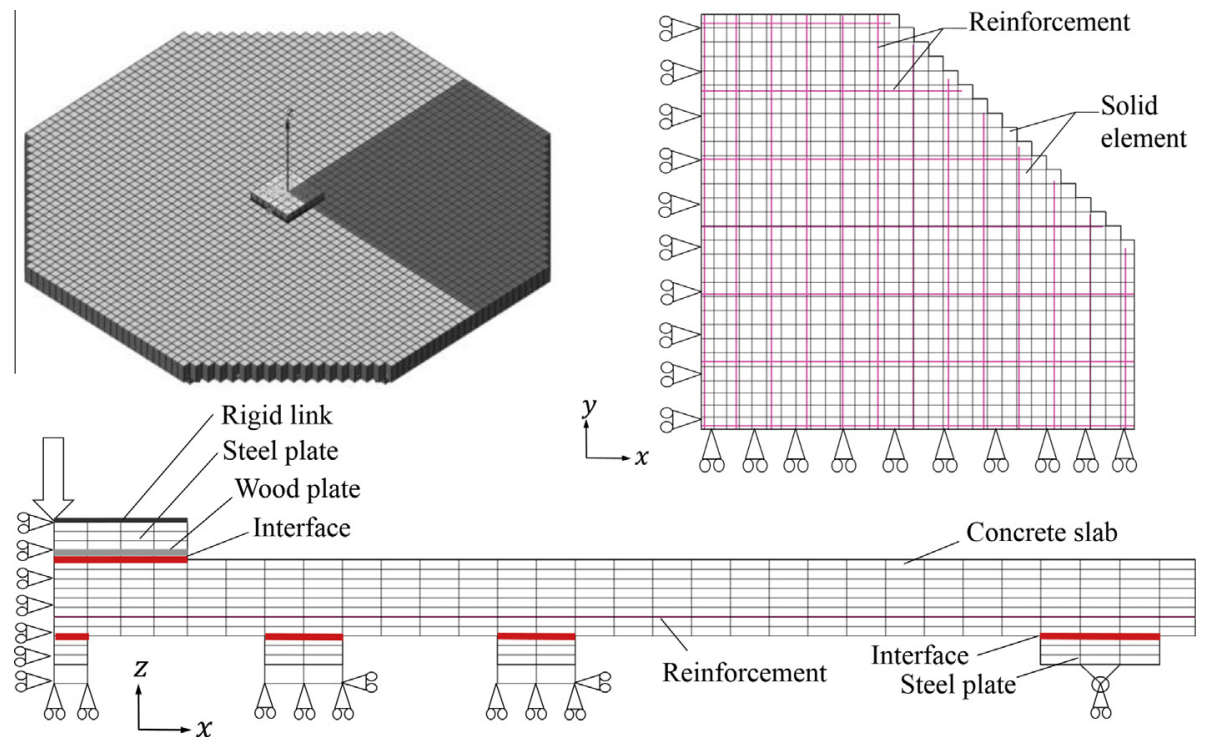

Fig. 3. FE model of the tested slabs.

of 0.01 . Both material and geometric nonlinearity were included in the FE analysis.

To investigate the influence of variable modelling choices, parameters describing different modelling assumptions were taken into account. Several parameters describing geometric nonlinearity, element properties, and the modelling of concrete, reinforcement, and supports were selected to be studied, see
Table 1. The influence of geometric nonlinearity was included in this study due to large deflections observed during the tests. Element properties including element type, element order and element density were included to examine their influence on the model. Parameters regarding material model of concrete, such as crack band width and Poisson's ratio, were included to study the effect of modelling recommendations found in the literature. The 
Table 1

Parametric study: the shaded alternatives were used for reference model and others are used for comparative model.

\begin{tabular}{|c|c|c|}
\hline Geometric nonlinearity & $\begin{array}{l}\text { Included } \\
\text { Excluded }\end{array}$ & \\
\hline Element properties & $\begin{array}{l}\text { Element type } \\
\text { Element } \\
\text { order } \\
\text { Element } \\
\text { density }\end{array}$ & $\begin{array}{l}\text { Brick } \\
\text { Wedge } \\
\text { First order } \\
\text { Second order } \\
\text { Coarse } \\
\text { Medium } \\
\text { Dense }\end{array}$ \\
\hline Modelling of concrete & $\begin{array}{l}\text { Crack band } \\
\text { width } \\
\text { Poisson's } \\
\text { ratio }\end{array}$ & $\begin{array}{l}\text { Mean crack } \\
\text { distance } \\
\text { Element size } \\
\text { No reduction } \\
\text { Reduction }\end{array}$ \\
\hline Modelling of reinforcement & $\begin{array}{l}\text { Bar } \\
\text { Grid layer }\end{array}$ & $\begin{array}{l}\text { Full bond } \\
\text { Bond-slip } \\
\text { Full bond }\end{array}$ \\
\hline $\begin{array}{l}\text { Modelling of supports: Stiffness of } \\
\text { interface element }\end{array}$ & \multicolumn{2}{|c|}{$\begin{array}{l}\text { Based on material property only } \\
\text { Based on material property and } \\
\text { deflection of pipe roller } \\
\text { Calibrated based on the test } \\
\text { result at elastic stage }\end{array}$} \\
\hline
\end{tabular}

approaches to model reinforcement as grid layers or as bars; the interaction with or without bond-slip between reinforcement and the concrete were included to study their effect on analysis results. The normal stiffness of the interface layers at the supports was varied to study the influence of support stiffness on the load distribution. A large number of initial analyses of varying modelling choices were carried out. The combination of alternatives that appeared to be best and efficient were chosen as a reference model, see shaded alternatives in Table 1 . The influence of alternative modelling choices was studied through comparative models; all input data but the studied parameter was kept the same in the comparative and the reference models. In total nine analyses were carried out, varying one parameter at a time.

\subsection{Geometric nonlinearity}

In the reference model the geometric nonlinearity with the Total Lagrange description [15] was included whereas in the comparative model the geometric nonlinearity was excluded. In the analysis of concrete structures including non-linear material, linear geometry is often a sufficient assumption, i.e. the equilibrium equations are based on the un-deformed geometry and the strains are linear functions of the nodal displacements. This assumption limits the applicability of the analysis to minor displacements, rotations and strains. For the structure studied, the displacement has to be considered "large" since it even exceeds the thickness of the slab; accordingly $[15,20]$, geometric nonlinearity needs to be included in the reference model. In this context, the GreenLagrange strain and 2nd Piola-Kirchhoff stress were adopted in the equilibrium equations and the large deformation was accounted for. In the comparative model, geometrical nonlinearity was excluded to witness the difference in response.

\subsection{Element properties}

The parametric study of element properties included models that had different element shapes, different element orders and different element sizes. The properties of the parameters studied for the reference and comparative models are shown in Table 2. First order eight-node brick elements, $40 \times 40 \times 10 \mathrm{~mm}^{3}$ (length $\times$ width $\times$ height), was used in the reference model. As this
Table 2

Varying modelling choices regarding element properties for the reference and comparative models.

\begin{tabular}{lllll}
\hline Model & $\begin{array}{l}\text { Element } \\
\text { shape }\end{array}$ & Element size $(\mathrm{mm})$ & $\begin{array}{l}\text { Element } \\
\text { order }\end{array}$ & Aspect ratio \\
\hline Reference & Brick & $40 \times 40 \times 10$ (Coarse) & First order & $1: 1: 0.250$ \\
B30I & Brick & $30 \times 30 \times 9$ (Medium) & First order & $1: 1: 0.296$ \\
B20I & Brick & $20 \times 20 \times 8$ (Dense) & First order & $1: 1: 0.400$ \\
W40I & Wedge & $40 \times 40 \times 10$ & First order & $1: 1: 0.250$ \\
B40II & Brick & $40 \times 40 \times 27$ & Second order & $1: 1: 0.667$ \\
\hline
\end{tabular}

element type is rather poor in describing bending due to shear locking, a convergence study was conducted, which showed that at least eight element layers over the thickness were needed. This requirement made the aspect ratio very small (only 0.25 ) but still larger than minimum value $(=0.12)$ [15]. To study the influence of mesh density, $30 \times 30 \times 9 \mathrm{~mm}$ and $20 \times 20 \times 8 \mathrm{~mm}$ were chosen for comparative model B30I and B20I, respectively. B30I means first order brick element with a size of $30 \mathrm{~mm}$ in plane. In comparative model W40I, wedge elements were chosen to investigate the influence of element shape. Due to the difficulties of achieving converging solutions given tetrahedral elements, a comparative model using these elements was excluded. In comparative model B40II, second order elements were used to study the possibility of reducing both model size and computation time. In this model, the number of element layers in the thickness direction was decreased to three layers.

\subsection{Material model of concrete}

The concrete was modelled based on the Total Strain Rotating Crack Model [15]. The constitutive model has originally been developed by Vecchio \& Collins [21]; the three-dimensional extension of this theory was established by Selby \& Vecchio [13]. In tension, a smeared rotating crack model was used. In this approach, the crack width $w$ is related to the crack strain $\varepsilon_{c r}$ perpendicular to the crack via a characteristic length - the crack band width $h b$. The advantage is that the formulation remains local and the algorithmic structure of the finite element code only requires minor adjustments, limited to the part of the code responsible for evaluating of the stress (and stiffness) corresponding to a given strain increment [11]. For unreinforced concrete, the crack band width is typically chosen as one element length [11]. For reinforced concrete, when the reinforcement is modelled assuming complete interaction with the surrounding concrete, the distribution of one crack is rather smeared over the mean crack distance instead [22]. Fig. 4 (left) shows the tensile property of concrete for the reference model. On the other hand, when slip is allowed between the reinforcement and the concrete, the crack band width is assumed to be approximately the size of a row of element, which the crack can be localized.

The behaviour of concrete in compression was described by an isotropic damage constitutive law. When the stress-strain relationship is used in numerical analyses, the localization of deformation in compressive failure needs to be taken into account. The compression softening behaviour is related to the boundary conditions and size of the specimen [23]. Consequently, as the stressstrain relation by Thorenfeldt et al. [24] has been calibrated by measurements of a compression test on $300 \mathrm{~mm}$ long cylinders, the softening branch needs to be modified for the concrete element size used in the FE model. Thus, the stress-strain curve according to Thorenfeldt was modified for the concrete element size as suggested by Zandi et al. [25], without considering the reduction due to lateral crack; see Fig. 4 (right). 

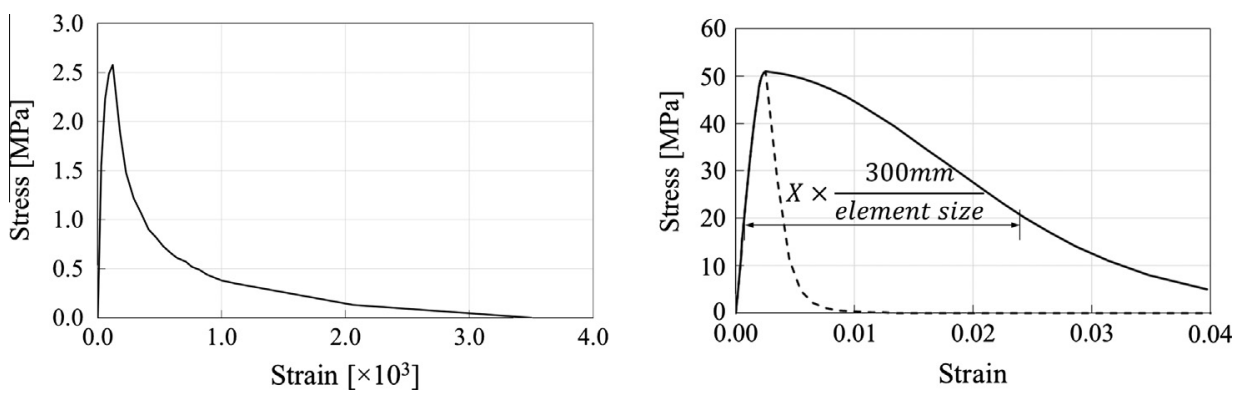

Fig. 4. Input for tensile (left) and compressive (right) models of concrete for reference model.

The other material properties has been estimated based on the tested compressive strength, according to Model Code 90 [26]; see Table 3.

The parameter study included analyses of varying crack band widths and reductions of Poisson's ratio, as in Table 4. The table also gives features of analyses based on reference model, model CEPU and CPPR. In the reference model, the crack band width was assumed to be equal to the mean crack distance, and a constant Poisson's ratio as for un-cracked concrete was used. The mean crack distance was measured based on the experiments to be around $100 \mathrm{~mm}$. In comparative model CEPU, the crack band width was chosen as the element size in the plane of the slab ( $40 \mathrm{~mm}$ for reference model) to study the influence on crack localisation and load-deflection response. In comparative model CPPR, Poisson's ratio was reduced after cracking to account for that cracked concrete have lower lateral contraction than elastic material, as reported by Selby and Vecchio [13]. In a fully cracked state, the Poisson's ratio was reduced to zero.

\subsection{Modelling of reinforcement}

The interaction between reinforcement and concrete can be modelled as fully bonded or by introducing a bond-slip relation. In DIANA, fully bonded reinforcement adds stiffness to the concrete finite elements but it does not have independent degrees of freedom. The space occupied by the reinforcement is ignored and does not contribute to the weight of the element. The fully bonded reinforcement in a slab can be modelled as grid layers or as individual bars, exemplified by the slab studied in Fig. 5 . The thickness of each grid layer is calculated as the total reinforcement cross-sectional area for a unit width of the slab divided by the unit width. The amount of reinforcement for each reinforcement layer

Table 3

Material parameters of concrete.

\begin{tabular}{ll}
\hline Parameter & Value \\
\hline Elastic modulus (MPa) & $E_{c}=24.5$ \\
Poisson's ratio & $v=0.15$ \\
Compressive strength $(\mathrm{MPa})$ & $f_{c m}=50.9$ \\
Strain at $f_{c m}$ & $\varepsilon_{c 1}=-0.0022$ \\
Tensile strength $(\mathrm{MPa})$ & $f_{c t m}=2.7$ \\
Fracture energy $\left(\mathrm{Nm} / \mathrm{m}^{2}\right)$ & $G_{f}=127$ \\
Crack bandwidth $(\mathrm{m})$ & $h_{b}=0.085$ \\
\hline
\end{tabular}

Table 4

Three models regarding concrete modelling.

\begin{tabular}{lll}
\hline Models & Crack band width & Reduction of Poisson's ratio \\
\hline Reference & Mean crack distance & No reduction \\
CEPU & Element size & No reduction \\
CPPR & Mean crack distance & Reduction \\
\hline
\end{tabular}

is defined separately and adds stiffness in the direction of the reinforcement bars separately.

In the analyses of the slab, the material property of the reinforcement was described by a Von Mises plasticity model, including strain hardening, using values obtained from material tests; see Fig. 6.

The parametric study included different approaches to model the reinforcement and the interaction between reinforcement and concrete. The properties of the analyses based on reference model, GLFB and TBBS are shown in Table 5. In the reference model, reinforcement was modelled as fully bonded individual
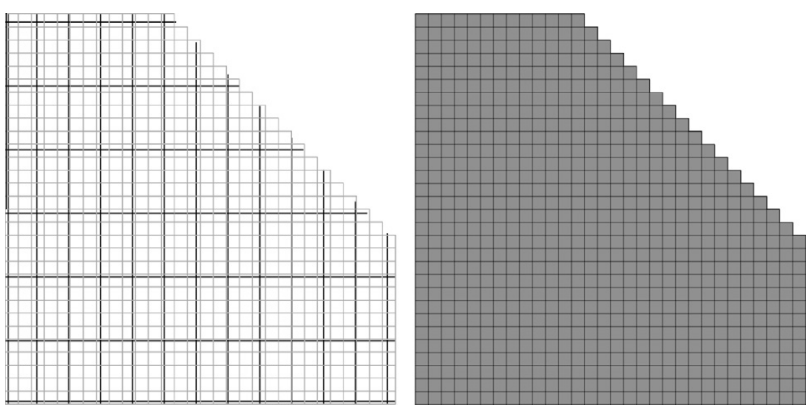

Fig. 5. Truss bar reinforcement (left) and grid layer reinforcement (right) for the RC slab model.

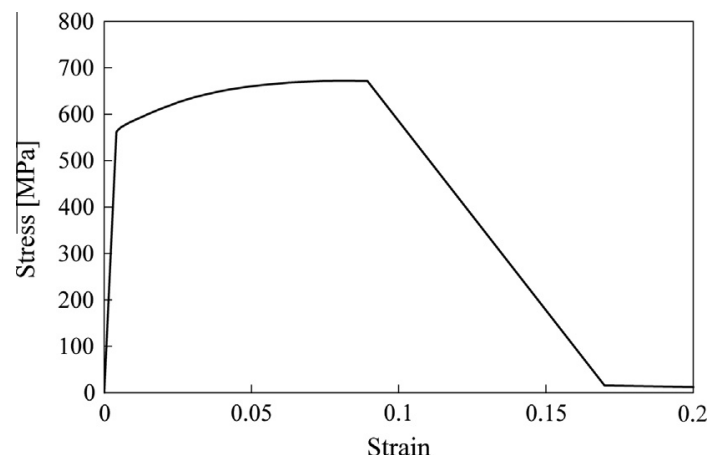

Fig. 6. Input for material model of reinforcement steel.

Table 5

Three models regarding reinforcement modelling.

\begin{tabular}{lll}
\hline Models & Reinforcement layer & Interaction between R\&C \\
\hline Reference & Bar & Full bond \\
GLFB & Grid layer & Full bond \\
TBBS & Bar & Bond-slip \\
\hline
\end{tabular}


bars. In comparative model GLFB, an embedded grid layer was instead used to model the fully bonded reinforcement. In comparative model TBBS, interface elements describing a bond-slip relation were used to investigate the influence of interaction between concrete and reinforcement.

When the bond-slip model was used, an option in the software to add bond-slip to the embedded reinforcement was used. This option was pre-processed by the software so that two-node truss elements for the reinforcement were connected to the concrete elements by line interface elements. These interface elements described a bond-slip behaviour in terms of a relationship between the shear traction and the relative displacement along the bars. An analytical bond-slip relation for unconfined concrete under "good" bond conditions given in the CEB-FIP Model Code [26] was assumed; see Fig. 7 (left). That is, given the notation used in Model Code (1990), $s_{1}=s_{2}=0.6 \mathrm{~mm}, s_{3}=1.0 \mathrm{~mm}$, $\tau_{\max }=14.26 \mathrm{MPa}$ and $\tau_{f}=2.14 \mathrm{MPa}$.

\subsection{Modelling of supports}

The support system in the test consist steel plates and rollers, see Fig. 2 (left). In the FE model, each support was modelled using a steel plate and plane quadrilateral interface elements $(4+4$ nodes) between the steel plate and the concrete; see Fig. 3 (bottom). In this way, the stiffness of the support system could be reflected in the stiffness of the interface elements. The response of the interface material was based on the Mohr-Coulomb friction criterion [27] with tension cut-off; see Fig. 7 (right). With this model, only compression was allowed normal to the interface plane.

To investigate how the distribution of the support reaction was affected by the stiffness of the support system, a parametric study with varying support stiffness was carried out. The parametric study included the reference model and two comparative models, DR and SDR; see Table 6. Three values of normal stiffness of the interface element were used in this study. A high stiffness, $1.0 \times 1013 \mathrm{~N} / \mathrm{m}^{3}$, was selected in the reference model, reflecting the contact between the concrete and the steel plate alone. In the model DR, the normal stiffness was calculated based on the deformation of the roller in the elastic range. The value in the model SDR was chosen based on the calibration of analysed reaction distributions to the measured distribution from tests in the elastic range.

\section{Results and discussion}

As mentioned, the geometry and test set-up were designed so that the RC slabs tested were expected to fail in bending due to yielding of the reinforcement. The three slabs tested showed very similar results. In the tests, cracking started when the load was
Table 6

Three models regarding normal stiffness of interface element.

\begin{tabular}{ll}
\hline Models & Normal stiffness $\left(\mathrm{N} / \mathrm{m}^{3}\right)$ \\
\hline Reference & $1.0 \times 10^{13}$ \\
DR & $4.5 \times 10^{9}$ \\
SDR & $1.2 \times 10^{9}$ \\
\hline
\end{tabular}

$27.4 \mathrm{kN}$ ( $\mathrm{std}=0.5$ ) on average, followed by bending hardening. The slabs failed at the ultimate load of $69.8 \mathrm{kN}$ ( $\mathrm{std}=1.1$ ) on average with a rupture of reinforcement bars; the tests were aborted when two bars had ruptured [19]. In the following section, the load-deflection response, crack pattern, and reaction force distribution from the analyses are compared to the test results.

\subsection{Load-deflection}

Fig. 8 shows the load-deflection relations obtained, both from the FE analysis and test. Each figure shows a comparison of results when varying one parameter. The results from the test and the reference FE analysis are included in all diagrams and demonstrate reasonable agreement. Before the cracking load reached, the response from both test and analyses illustrates an elastic behaviour. After cracking, a clear bending hardening behaviour followed. The ultimate capacity of the slab was reached when the deflection at the centre of the slab was almost $120 \mathrm{~mm}$. Comparing the results of the reference FE analysis to the experimental results, the cracking load was overestimated whereas the ultimate load was underestimated by the analysis. The cracking load in the analysis was $32 \mathrm{kN}$, which was $16.7 \%$ higher than that from the experiment $(27.4 \mathrm{kN})$. One reason for this difference is that, in the analysis, cracking did not took place until the tensile strength was reached at the bottom integration point of the slab; as this point was situated $5 \mathrm{~mm}$ from the bottom, a higher load could be applied before the tensile strength was reached here. In the analysis, the ultimate load was $65.3 \mathrm{kN}$, which was $6.4 \%$ lower than that in the test $(69.8 \mathrm{kN})$. The numerical analysis of the reference model stopped due to lack of convergence when the reinforcement stress decreased after ultimate stress was reached (see Fig. 6), showing that the slab failed due to the yielding of reinforcement. This fact agrees with the observations from the experiment.

Fig. 8a indicates that geometric nonlinearity has a substantial effect on the load-carrying capacity of the slab. Compared to the load deflection response with linear geometry, the response with geometric nonlinearity is much closer to the experimental result for large deflections and regarding the ultimate capacity. For deflections larger than the slab thickness, part of the load is counteracted by the development of radial tensile stresses in combination with a compressive ring around the slab edge, commonly called the "membrane effect" [8]; neglecting this effect leads to an underestimation of the load-carrying capacity.
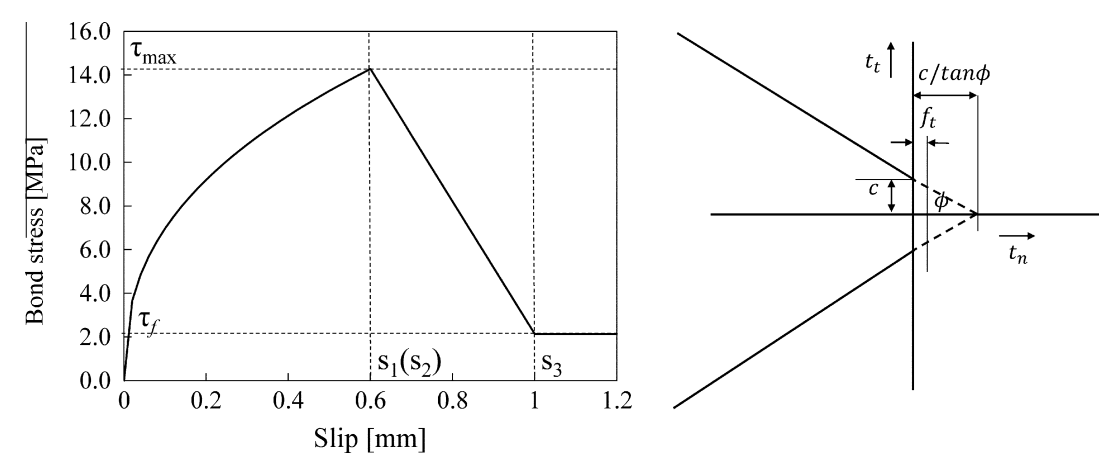

Fig. 7. Bond versus slip assumed for the interaction between concrete and reinforcement (left); Mohr-Coulomb friction criterion [27] (right). 

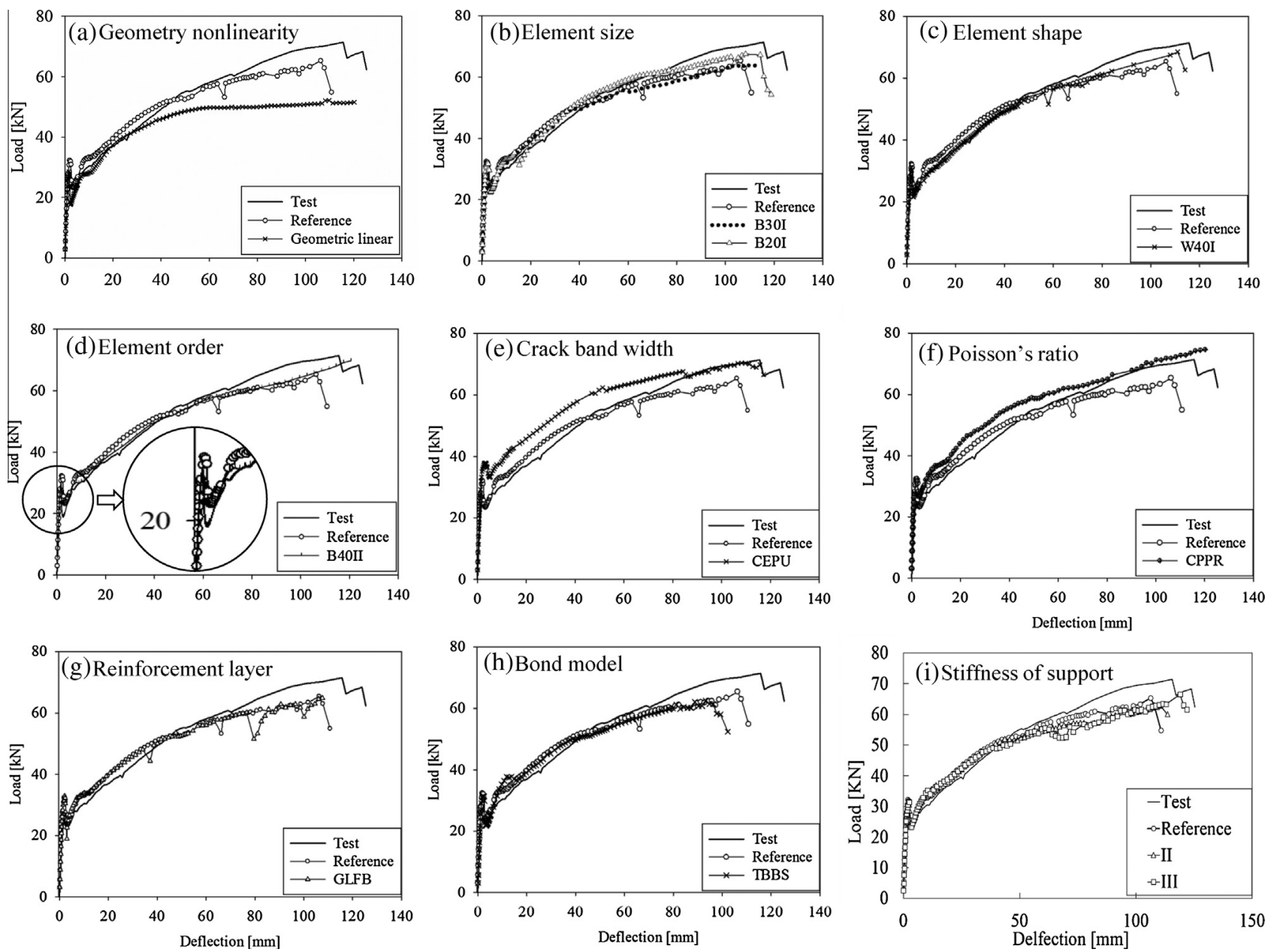

Fig. 8. Load versus mid-span deflection.

Fig. 8b-d present results with regard to element size, element shape and element order, respectively. Comparing the load deflection responses of the models with different element sizes to the reference model in Fig. 8b, they were generally close due to same crack bandwidth, but the comparative model B20I had a response closer to the test because of the denser mesh. Concerning the study of element shapes in Fig. 8c, the load deflection response of the analysis with wedge elements was in better agreement with the test result than that from the reference analysis, but it was more difficult to reach convergence. Regarding element order, see Fig. 8d, the 20-nodes second order element model, which was supposed to have better bending behaviour, presented a similar load deflection response as the reference model with first order elements. This indicates that eight layers of first order elements in the thickness direction were enough to describe bending behaviour. However, the model with first order elements has a larger model size costing longer computation time, which must be considered during modelling in engineering practice.

Fig. 8e and f present analyses results with regard to the modelling of concrete. From the results displayed in Fig. 8e, the choice of crack band width plays an important role to the load-carrying capacity of the RC slab. Comparing the load deflection curve, the model with the crack band width equal to the element size have too high a capacity. This is reasonable because the smaller crack band width leads to higher consumption of energy if the analyses shows the same crack pattern. From the figures of crack patterns (Fig. 9b and g) later in the paper, cracks in the analyses did not localize in single element rows. Instead, both the crack patterns are somewhat diffuse and the rows of cracked elements are interconnected along the cracks. Concerning Poisson's ratio in Fig. 8f, the load deflection curve from the analysis with reduced Poisson's ratio shows a closer capacity but a bit higher stiffness than that of the reference model and experiment. This was reasonable because when Poisson's ratio decreases during crack formation, the parts of the slab subjected to tension stop to contract in the perpendicular direction, which decreases the size of tensile cracks, leading to higher capacity. However, such results coming from this analysis are dependent on the effectiveness of the crack models implemented in this software (DIANA).

Fig. $8 \mathrm{~g}$ and $\mathrm{h}$ present results of analysis with regard to the modelling of reinforcement. Fig. $8 \mathrm{~g}$ presents comparison between the model with grid layer reinforcement and the reference model with bar reinforcement. The general shapes of the load deflection curves are similar. The different approaches to model reinforcement did not change the reinforcement ratio, thus not the bending capacity, nor did it change the load-deflection response of the slab. Furthermore, Fig. 8h shows that the load-carrying capacity and the load-deflection response were not influenced by the interaction between reinforcement and concrete either.

Fig. $8 \mathrm{i}$ present results of analysis with regard to modelling of support stiffness. The overall response of the slab in terms of the load-deflection relation was similar in the reference and comparative models. However, the comparative models, with considerably lower normal stiffness of the supports, showed a somewhat higher ductility due to decreased overall stiffness. 


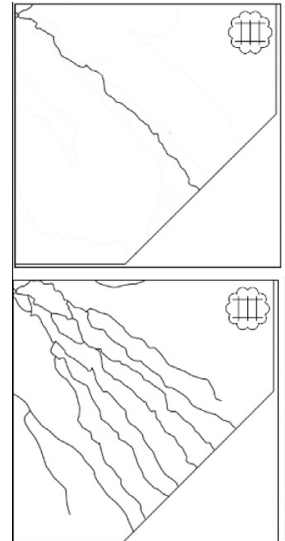

(a) Test
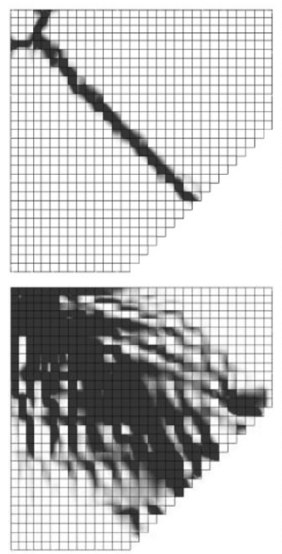

(f) B40II
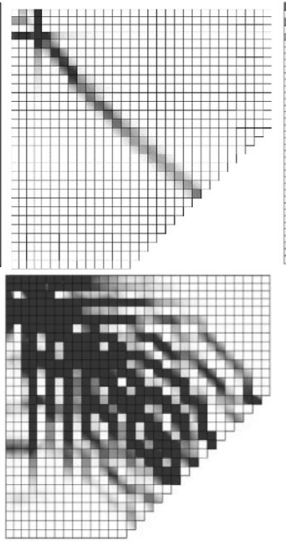

(b) Reference
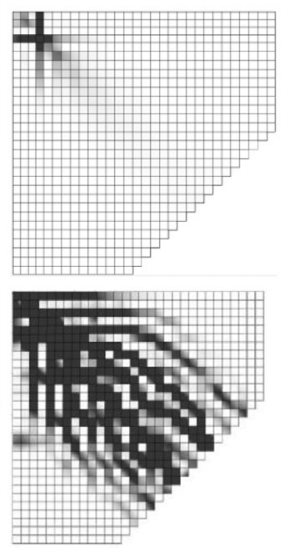

(g) CEPU
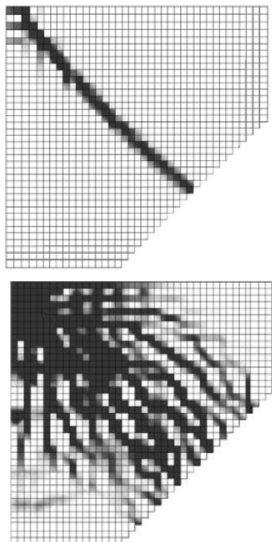

(c) B30I
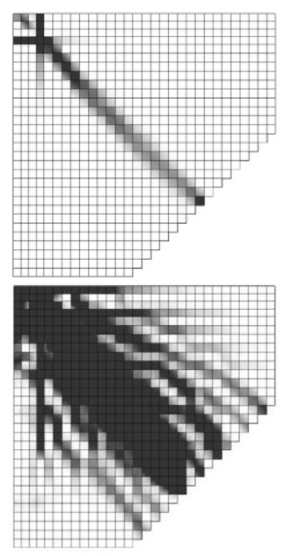

(h) CPPR

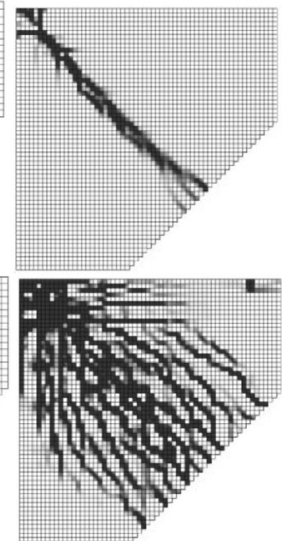

(d) B20I
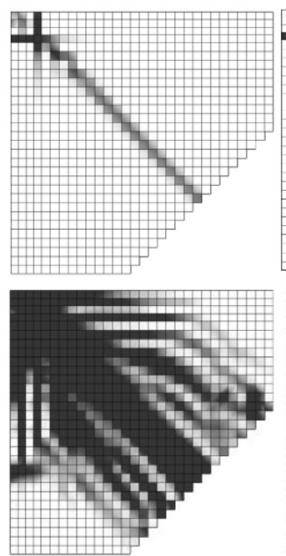

(i) GLFB
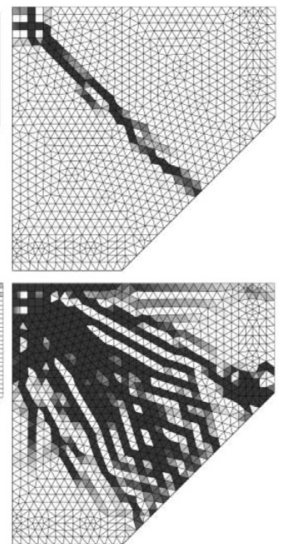

(e) W40I
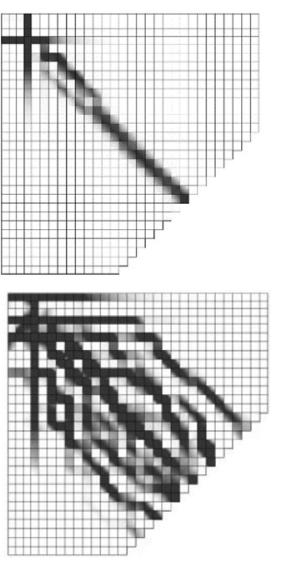

(j) TBBS

Fig. 9. Crack pattern at stage A (initial crack) and stage C (fully cracked stage).

\subsection{Crack pattern}

Both analyses and experiments resulted in bending cracks and bending failure. In Fig. 9, sketches of the initial and final crack patterns at the bottom of the slab are shown, both for the slab tested (CR1) and the FE analyses at stage A (initial crack stage, deflection $=2.5 \mathrm{~mm}$ ) in the first row and at stage $C$ (fully crack stage, deflection $=100 \mathrm{~mm}$ ) in the second row. The sketch of the test results (Fig. 9a) does not indicate the crack width, merely the number and directions of the cracks. In the strain based crack pattern resulting from $\mathrm{FE}$ analysis, the black colour $\left(\varepsilon>5 \times 10^{-3}\right.$ for Fig. $9 \mathrm{~b}-\mathrm{f}$ and $\mathrm{h}-\mathrm{j}$, and $\varepsilon>0.01$ for Fig. $9 \mathrm{~g}$ ) represents fully open cracks and the grey colour represents minor crack widths. In the reference model, see Fig. 9b, the initial cracks appeared at the end of the elastic stage reaching from the centre of the slab diagonally towards the unsupported edge, similarly as in the tests. These cracks were also the widest cracks when the maximum load was approached. Compared to the tests, the analysis based on the reference model (Fig. 9b) gave a reasonable prediction of the crack pattern when the slab was loaded. Even though the crack extended in a zig-zag pattern along the diagonal line of the slab, the cracking still showed only a minor tendency to form along the mesh line. This phenomenon was also verified in the parameter study regarding varying element shapes. It was also observed that due to the tying of all the nodes at the top of the steel plate, the crack pattern follows the steel plate edges. The number of cracks at the final stage $C$ could not be predicted exactly in the reference model. As shown in Fig. 9b, there were totally 10 main cracks at stage $C$ in the pattern from the test, whilst only six distinct and separate cracks could be distinguished in the analysis.

Fig. 9c-f present results with regard to varied element properties. By comparing the crack pattern of the models with different mesh densities, see Fig. 9b-d, cracks became easier to recognize when the element size was decreased. Also, the number of separate cracks increased and became more similar to that in the test. Concerning the study of element shape, see Fig. 9b and e, it was observed that the element shape influenced the crack pattern without influencing the direction of crack propagation in general. From the crack pattern in Fig. 9b and f, it can be seen that a somewhat more diffused crack pattern was obtained with higher order elements. For second order elements, the cracks at stage A; see Fig. 9f, did not propagate in a zig-zag pattern or along mesh lines as for first order elements, see Fig. 9a. This is because the middle nodes in the elements make it possible for crack strain to vary within the elements. At stage $C$ the cracks are more difficult to recognize using second order elements compared to the reference model because of the number of element in thickness direction. In the reference model, there were eight elements in total in the thickness direction and the reinforcement was placed between the second and third layers from the bottom. This allowed the elements in the first layer to crack individually. However, in comparative model B40II, there were only three layers of elements in the thickness direction. The reinforcement was placed within the bottom layer of concrete elements; see Fig. 10. Thus, the cracks had to 


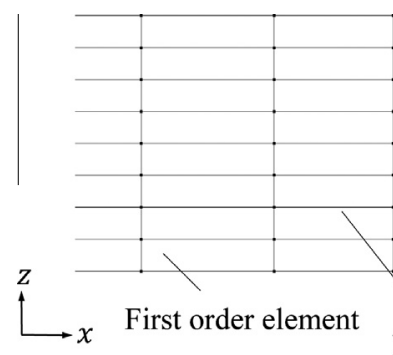

Fig. 10. The cross-section of first and second order element.

distribute to all concrete elements along the reinforcement bars since they were fully bonded.

Fig. $9 \mathrm{~g}$ and h present analyses results with regard to the modelling of concrete. From the results displayed in Fig. 9b and g, it can be seen that the choice of crack band width plays a major role for the crack pattern. Comparing the crack pattern of the different models at stage $A$, the initial crack in the model with the crack band width equal to the element size did not appear at the same displacement level as in the reference model, because it took more energy to form the first crack when a smaller crack band width was used. In Fig. 9b and h, the model with reduced Poisson's ratio gave less localization of cracks at stage $C$ compared to the reference model. This is because after cracking, the high Poisson's ratio makes the crack becomes wider and deeper, whereas, when zero Poisson's ratio is used after cracking, more distributed cracks happens to the elements.

Fig. 9i and j present analyses results with regard to the modelling of reinforcement. Fig. 9i present the crack pattern for the model with grid layer reinforcement. As observed at stage $C$, the crack pattern was more evenly distributed compared to that from reference analysis; see Fig. $11 \mathrm{~b}$. This result is understandable because with grid layer reinforcement, all the concrete elements at the reinforcement level had added stiffness from the grid layer, which made the stiffness of the slab evenly distributed in contrast to the reference model which had separate reinforcement bars. The comparison between Fig. 9b and j showed that the analysis including a bond-slip relation for the reinforcement presented cracks that were more localized, compared to the reference model with fully bonded reinforcement.

\subsection{Load distribution}

In this section, the load distribution is investigated by comparing the results from the test, the reference analysis and the parametric study. There are two aspects of interest: the load distribution between the two main directions, and the load distribution along the supports on each side of the slab. These two aspects are discussed below.

\subsubsection{Reaction forces in the two main directions}

The influence of the different reinforcement ratios on the reaction forces in the two main directions was studied. In Fig. 11 (left), the total reaction force at the supports in the strong (more reinforcement) and weak (less reinforcement) directions from the test as well as analysis are presented. Comparing the reaction force carried by the supports in the two directions, it could be observed that the load carried in the strong direction continued to increase upon cracking, whereas almost no increase in the support reaction in the weak direction was observed.

In the analysis of reference FE model, two-thirds of the total reaction force was carried in the strong direction at stage $C$. This results corresponds to the difference in reinforcement amounts; the reinforcement ratio in the strong direction was twice as high as in the weak direction. In the test, the proportion of the reaction force taken by the strong direction was even higher, up to $71 \%$ at stage $C$.

Fig. 11 also compares the reaction forces in the two main directions for varying support stiffness, i.e. for comparative models DR and SDR. When the normal stiffness of the support interface layers was decreased, the differences between the comparative and reference models were minor in the weak direction but more evident in the strong direction. With decreased support stiffness, a smaller proportion of the load was carried in the strong direction and, as mentioned previously, decreasing the load-carrying capacity.

\subsubsection{Reaction distribution along the support edges}

In Fig. 12(a) and (b) the crack pattern at stage $C$ and the load distribution along the supported edges are visualized at three stages. Here stage B (deflection $=30 \mathrm{~mm}$ ) is added, corresponding to the initial yielding of the reinforcement. In Fig. 12(b), the curves "West", "East", "North", and "South" indicate the results from the test. It can be seen that for both the tested and analysed slab, the reaction force was more evenly distributed over the supports in the strong direction (North, South) than in the weak direction. This finding agrees with what could be expected as the denser reinforcement perpendicular to the support performs better to transfer load to support after crack. In the tests, it was observed that cracks extended perpendicularly to the supports in the strong direction. These cracks occurred also in the FE analysis, but did not extend all the way to the support. These cracks seem to affect the distribution of reaction forces; in the strong direction, the middle support (no. 3) carried less loads than adjacent supports.

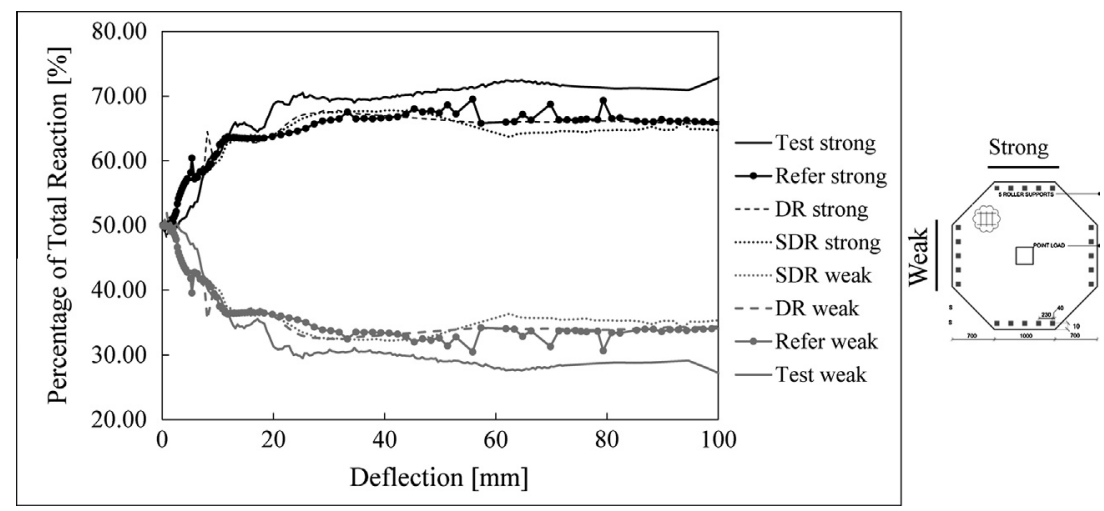

Fig. 11. Reaction force distribution in two main directions. 


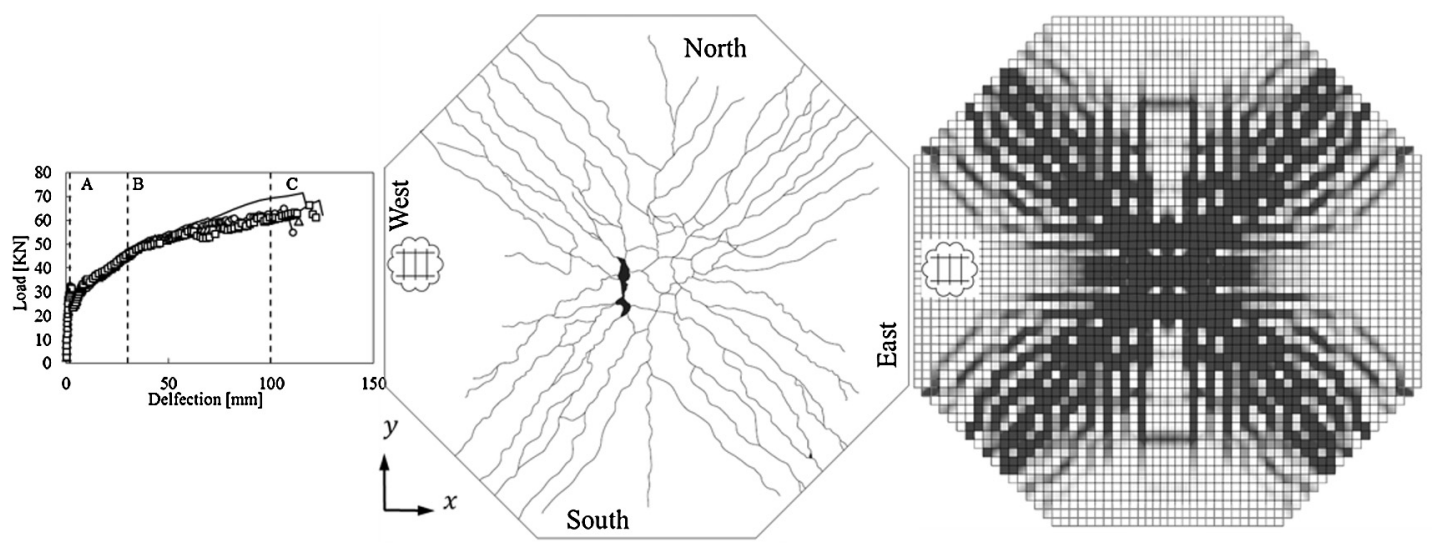

(a)

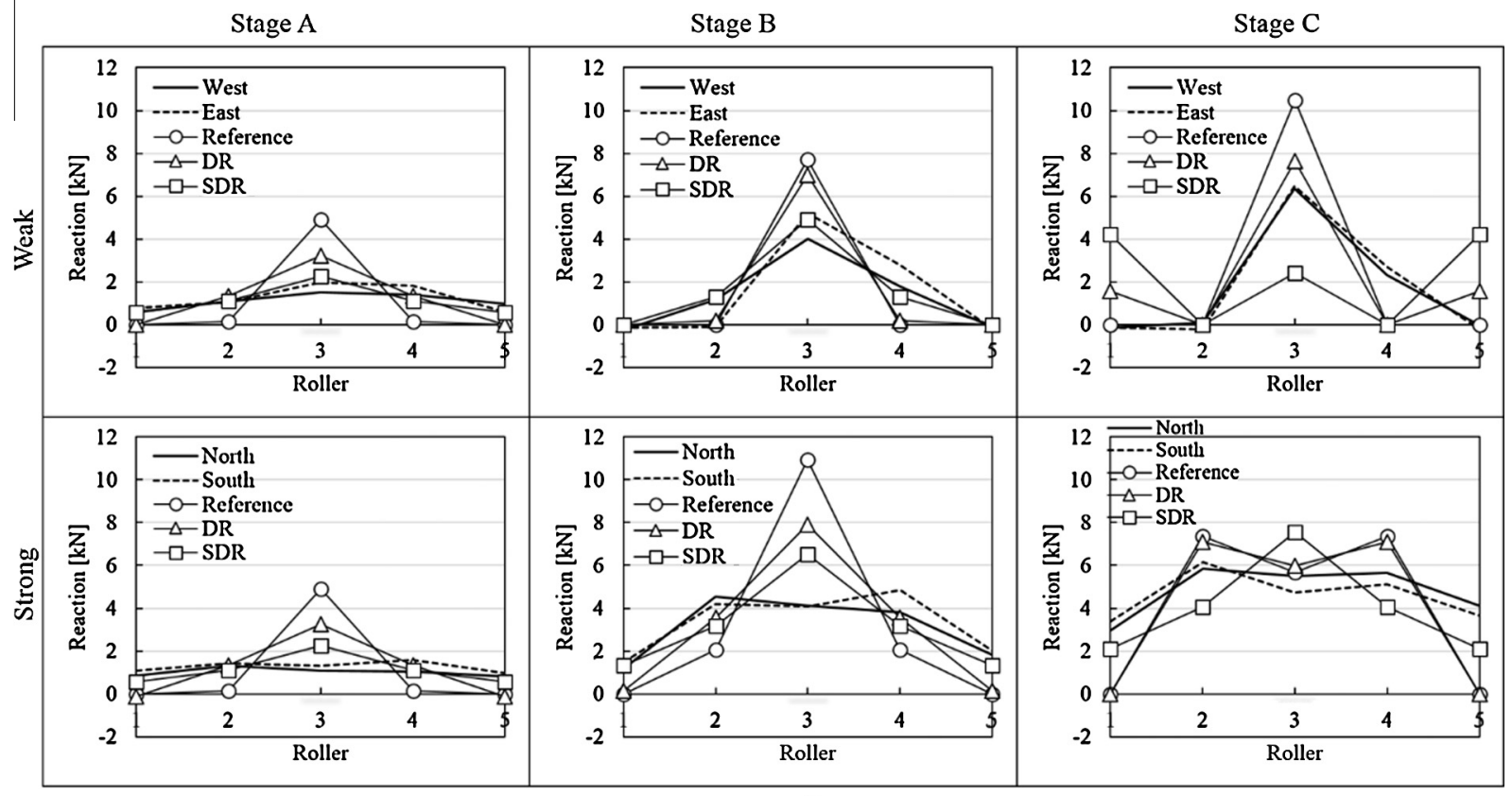

(b)

Fig. 12. Crack pattern and reaction force distribution over the support edge.

In comparing the results from tests and $\mathrm{FE}$ analyses, it is observed that the comparative model SDR, with the weakest support stiffness, gave a reasonably good estimation of the distribution of reaction force along the slab edges at stages $\mathrm{A}$ and $\mathrm{B}$. Especially for supports in the weak direction, the middle roller (no. 3) obtained the highest reaction, followed by the adjacent rollers (nos. 2 and 4). The outermost rollers (nos. 1 and 5) obtained almost no reaction force. The reaction force distribution for the supports in the strong direction was different: the reaction force in the tests was more evenly distributed than in the FE analysis. The difference in load distribution between the test and FE analysis may be attributed to differences in structural response. At the initial crack stage, the material in the FE model displayed a more idealized elastic behaviour than the material in reality; when the slab was deformed, an equilibrating peripheral ring (see Fig. 13) was formed so that only the centre support rollers along the slab edges were able to support the slab. The difference at the fully cracked stage (C) may be attributed to the influence of the crack extending to the middle rollers in the strong direction in the tests, but not during the FE analysis. The occurrence of this crack seems to have reduced the load transfer to the middle roller (no. 3).

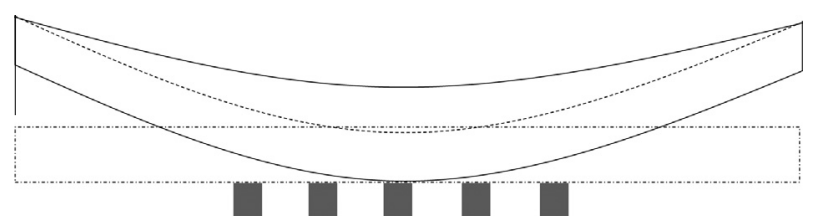

Fig. 13. Visualization of equilibrating peripheral ring.

The normal stiffness of the supports was found to be decisive for reflecting the reaction force distribution along the support edges. In the reference model, only the contact between the concrete and the steel plate was taken into account. In the model $\mathrm{DR}$, the deformation of the pipe rollers was included in the normal stiffness. In the model SDR, the deflection of the entire support system was accounted for. Here, the normal stiffness of the interface elements at the supports was calibrated by comparing the reaction force distribution to the tests in the elastic range. The study shows that the stiffness calibrated from the elastic response used in the model SDR was the optimal choice, revealing that the stiffness of the support system strongly influences the reaction distribution for RC slabs. 


\section{Conclusions}

The purpose of this study is made to investigate how accurately a nonlinear three-dimensional FE analysis using solid element can predict the structural behaviour of two-way RC slabs subjected to bending, as well as how different modelling choices influence analysis results. The load-carrying capacity, load-deflection response, crack pattern, and reaction force distribution were compared to experimental results. In order to develop modelling strategies for two-way slabs for practical use, an investigation including a reference analysis and several comparative analyses with varying parameters was carried out. However, the conclusions summarized below are proved to be valid for slab subjected to bending failure. Different conclusions could arise for slabs failing due to shear or punching.

By comparing the results of the FE analysis with test results, it was concluded that the reference model was capable of predicting the capacity, crack pattern and load distribution of the two-way slabs with reasonable accuracy. Both the initial cracking load and the ultimate load could be predicted within a reasonable error. The model was also capable of describing the crack pattern both at the initial and ultimate stages, but was unable to predict the exact number of cracks in the ultimate stage. The analysis correctly described the reaction force distribution between the two main directions, in proportion to the reinforcement amount in the different directions. However, the reaction force distribution along the support edges could be correctly described only after adjusting the vertical stiffness of the supports.

Through the parameter study, it can be concluded that the capacity predicted by the analysis of the two-way slab was significantly affected by such parameters as geometric nonlinearity, crack band width and Poisson's ratio. Geometric nonlinearity must be included when large deformations occur. The crack band width can be assumed to be the mean crack distance when fully bonded reinforcement is used. Assuming element size as crack band width resulted in an overestimation of the ultimate load. Using reduced Poisson' ratio led to closer results but a little overestimation. The crack pattern in the analysis was influenced by element size, element shape, element order, Poisson's ratio, whether the reinforcement was modelled with individual bars or as a grid, as well as whether full interaction or not was assumed between concrete and reinforcement. To clearly detect individual cracks, first order elements with dense mesh could be used, but this also brings longer computation time. Including a bond-slip relation for the interaction between reinforcement and concrete further improves the possibility of modelling the crack pattern in detail. Wedge element has the advantage of giving more freedom to the direction of crack propagation, which makes it a good choice if irregularly shaped structural components are difficult to mesh with brick elements. Second order elements and grid layer as reinforcement are good alternatives when the crack pattern is not of major interest. The reaction force distribution was found to be highly influenced by the stiffness of the supports. To estimate the support reaction distribution, the stiffness of the "support system" in reality, including e.g. columns, edge beams and transversal beams must be taken into account.

It could be concluded that the following modelling choices can be recommended to assess the structural behaviour of a two-way RC slab subjected to bending and modelled with 3D continuum finite elements: (a) geometric nonlinearity must be included if larger deflections than half the slab thickness may occur, (b) first order eight-node brick elements with at least eight elements over the cross-section height is sufficient, (c) the crack band width should be estimated as the mean crack distance if fully bonded reinforcement is used, (d) a reduction of Poisson's ratio for the concrete is not recommended if safety problem is considered, (e) fully bonded bar reinforcement is sufficient if a detailed simulation of the crack pattern would not be required and (f) the support stiffness needs to be included to describe support reaction distribution correctly, e.g. by using interface elements with a calibrated normal stiffness. If a detailed picture of the crack pattern is not needed, second order elements and embedded reinforcement grids are options by which the modelling and computation time may be saved.

\section{Acknowledgements}

The research presented was funded by the Swedish Transport Administration (Trafikverket) and the European Community's Seventh Framework Programme under Grant agreement NMP2-LA-2009-228663 (TailorCrete). More information about the research project TailorCrete can be found at www.tailorcrete.com.

\section{References}

[1] Plos M. Application of fracture mechanics to concrete bridges: finite element analyses and experiments. Gothenburg: Chalmers University of Technology; 1995.

[2] Sas G, Blanksvard T, Enochsson O, Taljsten B, Elfgren L. Photographic strain monitoring during full-scale failure testing of Ornskoldsvik bridge. Struct Heal Monit 2012;11(4):489-98.

[3] Sustainable Bridges. Guideline for load and resistance assessment of existing European railway bridges: advices on the use of advanced methods; 2007.

[4] CEB-FIP, fib Model Code for Concrete Structures 2010. Lausanne, Switzerland; 2013.

[5] Hendriks MAN, den Uijl JA, de Boer A, Feenstr PH, Belletti B, Damoni C. Guidelines for Nonlinear finite element analysis of concrete structures. Rijkswaterstaat Technisch Document RTD:1016:2012, Rijkswaterstaat Centre for Infrastructure; 2012.

[6] CEB-FIP. Fib bulletin no. 45: practitioners' guide to finite element modelling. Int Federation Struct Concr (fib); 2008.

[7] Jofriet C, McNiece M. Finite element analysis of reinforced concrete slabs. J Struct Div, ASCE 1971(97):785-806.

[8] Crisfield MA. Non-linear finite element analysis of solids and structures. 2. Advanced topic, vol. 1. John Wiley \& Sons; 1991.

[9] Rashid Y. Analysis of reinforced concrete pressure vessels. Nucl Eng Des $1968 ; 7: 334-44$

[10] Ngo D, Scordelis A. Finite element analysis of reinforced concrete beams. J Am Concr Inst 1967;64:152-63.

[11] Jirásek M, Bauer M. Numerical aspects of the crack band approach. Comput Struct 2012:110-111:60-78.

[12] Bazant ZP, Oh BH. Crack band theory for fracture of concrete, Chicago; 1983.

[13] Selby RG, Vecchio FJ. Three-dimensional constitutive relations for reinforced concrete, Toronto, Canada; 1993.

[14] den Uijl JA, Bigaj AJ. A bond model for ribbed bars based on concrete loaded in compression. Heron 1996;41:201-26.

[15] Diana TNO. Finite element analysis. User's Manual - Release 9.4.4. Delft, Netherlands; 2012.

[16] Lundgren K, Gylltoft K. A model for the bond between concrete and reinforcement. Mag Concr Res 2000;52(1):53-63.

[17] Lundgren K. Three-dimensional modelling of bond in reinforced concrete. Gothenburg: Chalmers University of Technology; 1999.

[18] Belletti B, Damoni C, Hendriks M, de Boer A. Analytical and numerical evaluation of the design shear resistance of reinforced concrete slabs. Struct Concr 2014.

[19] Fall D, Shu J, Rempling R, Lundgren K, Zandi K. Two-way slabs: experimental investigation of load redistributions in steel fibre reinforced concrete. Eng Struct 2014;80:61-74

[20] Crisfield MA. Non-linear finite element analysis of solids and structures, vol. 1. London: John Wiley \& Sons; 2000.

[21] Vecchio FJ, Collins MP. The modified compression field theory for reinforced concrete elements subjected to shear. ACI J 1986;83(22):219-31.

[22] Broo H. Shear and torsion in concrete structures. Chalmers University of Technology; 2008.

[23] Mier J. Strain-softening of concrete under multiaxial loading conditions. Eindhoven University of Technology; 1984.

[24] Thorenfeldt E, Tomaszewicz A, Jensen JJ. Mechanical properties of highstrength concrete and applications in design. Proc Symp Utiliz High-Strength Concr 1987.

[25] Hanjari KZ, Kettil P, Lundgren K. Modeling the structural behavior of frostdamaged reinforced concrete structures. Struct Infrastruct Eng 2010;9(5):416-31.

[26] CEB-FIP. Fib model code for concrete structures 1990, Lausanne, Switzerland; 1993.

[27] Vermeer PA, De Borst R. Non-associated plasticity for soils, concrete and rock. Delft, The Netherlands: Delft University of Technology; 1970. 